\title{
Basic framework for risk assessment for transgenic plants developed by the OECD: 20 years after the OECD "Blue Book"
}

\author{
Hans BERGMANS* \\ National Institute for Public Health and the Environment, GMO Office, PO Box 1, Bilthoven, 3720BA, The Netherlands
}

\section{INTRODUCTION}

The OECD publication "Recombinant DNA safety considerations" (OECD, 1986), better known as the "OECD Blue Book", has the reputation of being the first publication that endeavored to present comprehensive considerations on the environmental safety of recombinant DNA technology. Indeed, the book coined some of the key concepts in the discussion, e.g. the "case-by-case" and "stepby-step" approaches. But it also is a "snapshot in time", that illustrates the state-of-the-art of the biosafety discussion at that moment. As such, it has worked in an excellent way as a discussion starter that has sparked off a number of publications on the safety of the environmental use of transgenic plants and micro-organisms.

The Blue Book was initiated within OECD in 1983 as a follow-up to the OECD report "Biotechnology: International Trends and Perspectives" (Bull et al., 1983). The drafting of the Blue Book was entrusted to an ad hoc Committee of experts from academia, industry, and from governmental bodies. The impetus for OECD to look into matters of biosafety was the consideration that "recombinant DNA techniques have opened up new and promising possibilities in a wide range of applications and can be expected to bring considerable benefits to mankind" and that "a common understanding of the safety issues raised by recombinant DNA techniques will provide the basis for taking initial steps toward international consensus, the protection of health and the environment, the promotion of international commerce and the reduction of national barriers to trade in the field of biotechnology" (OECD, 1986).

So the Blue Book was to approach these biosafety issues in a positive spirit, based on an underlying belief that

*Corresponding author: hans.bergmans@ rivm.nl science-based discussions would lead to this common understanding.

This review will focus on the role of the Blue Book as a primer in the development of risk assessment of transgenic crops, and on how the science based discussion has developed further within OECD.

\section{THE OECD BLUE BOOK AND THE FIRST DEVELOPMENT OF ENVIRONMENTAL RISK ASSESSMENT OF TRANSGENIC PLANTS}

The mandate of the ad hoc drafting group was to "review country positions as to the safety in use of genetically engineered organisms at the industrial, agricultural and environmental levels, against the background of existing or planned legislation and regulations for the handling of micro-organisms"; and to "identify what criteria have been or may be adopted for the monitoring or authorization for production and use of genetically engineered organisms in industry, agriculture [and] the environment". Consequently, the safety considerations in the Blue Book are primarily derived from safety considerations that are specific to micro-organisms. When talking about agricultural and environmental safety concerns, the book first mentions that "recombinant DNA containing organisms will be developed for purposes involving uncontained applications in the environment, e.g. as pesticides, to improve plant growth, leach ore, enhance oil recovery and to degrade pollutants". Only in the second place it is considered "likely too that rDNA plants and animals will be used to enhance production of fiber, food and fodder. Other applications are likely to include production and use of certain animal and human drugs". 


\section{The Blue Book and risk assessment}

The methodology of risk assessment is treated summarily, as a "brief review of methods which are particularly relevant to the applications of rDNA micro-organisms. However, they also apply in principle to plants and animals". In fact, the section on risk assessment is not very straightforward. The thinking on risk assessment of genetically modified organisms had not yet crystallized out, but the main components are there. A case of environmental use of an rDNA containing (micro-)organism consists of (5) stages: (1) Formation (either deliberate or accidental) of the GMO; (2) Release (deliberate or accidental escape) into the environment; (3) Proliferation: "the subsequent multiplication, genetic reconstruction, growth, transport, modification and die-off of these micro-organisms in the environment, including possible transfer of genetic material to other micro-organisms"; (4) Establishment, including possible colonization of humans or other biota; and (5) Effect: "the subsequent occurrence of human or ecological effects due to interaction of the organism with some host or environmental factor". Stages (1), (2) and (5) were expected to be straightforward (e.g. stage (5) could be "analyzed by adapting conventional epidemiological or toxicological methods"; we would probably no longer be as confident). Stages (3) and (4) were expected to be more problematic, because of the key difficulty: the assessment of interactions of the microorganism with the existing ecosystem.

From these considerations it follows that the scientific framework for risk assessment is defined by "the difference between the properties of the modified organism and of the recipient organism". This difference can be predicted from knowledge about the traits coded by the donor sequences, and their expected effects in the physiological background of the recipient. Testing the new properties of the rDNA organism is feasible, because "the close similarity between the recipient and modified organism allows tests useful in describing the properties of the modified organism".

All this is still similar to the framework of risk assessment of contained use of GMOs in practice, although treatises on contained use seldom provide such a detailed description of the risk assessment methodology.

\section{Safety considerations associated with agricultural and environmental applications: the "stepwise" approach}

In the section on safety considerations associated with agricultural and environmental applications, the Blue Book takes a major step into the unknown: "Concern has been expressed that application of rDNA organisms in the environment may present ecological risks, and attempts have been made to evaluate this potential for harm. At this time, these attempts rest primarily on extrapolations from experiences with: (i) the introduction of naturally occurring organisms to ecosystems to which they are not native; (ii) evolution of novel traits in existing populations; and (iii) manipulation of agricultural crops, plantassociated microbes and animals. However, our predictive knowledge in some of these areas is not comparable to that developed for industrial applications and consequently these areas should be kept under review as the field develops."

For rDNA plants, the main safety concern mentioned is their potentially increased weediness, second is the potential for an inadvertent toxicity developed in the rDNA plant due to production of "a toxic secondary metabolite or protein toxin, particularly if the plant is engineered for resistance to an insect pest". An important conclusion is that these "safety concerns focus on whether environmental and agricultural applications of organisms modified by rDNA techniques pose an "incremental" risk. While rDNA techniques may result in the production of organisms expressing a combination of traits that are not observed in nature, genetic changes from rDNA techniques will often have inherently greater predictability compared to traditional techniques, because of the greater precision that the rDNA technique affords to particular modifications. It is expected that any risks associated with applications of rDNA organisms may be assessed in generally the same way as those associated with non-rDNA organisms. It is acknowledged that additional research and experience with rDNA micro-organisms, plants, and animals, should certainly increase our ability and precision to predict the outcome of introductions of rDNA organisms into the many varied ecosystems." This underpins one of the main conclusions of the Blue Book on the "stepwise approach": "the process of progressively decreasing physical containment, by which microorganisms and higher organisms are developed routinely for agricultural and environmental applications - i.e. research in the laboratory, research in microcosms and other contained environments, small field testing, and large field testing - allows a logical, incremental stepwise process whereby safety and performance data are collected. In this development process, the organism is characterized and carefully observed at each stage and a prediction can be made of its behavior in subsequent less confined stages of development."

In parenthesis it should be mentioned that the Blue Book does not really offer an underpinning for its other main principle: the case-by-case approach. It is postulated as the approach to be taken, and explained as follows: "Case-by-case means an individual review of a proposal against assessment criteria which are relevant to the particular proposal; this is not intended to imply 
that every case will require review by a national or other authority since various classes of proposals may be excluded."

\section{Recommendations}

The Blue Book summarizes its recommendations "with specific reference to agricultural and environmental applications" as follows: "a) use the existing considerable data on the environmental and human health effects of living organisms to guide risk assessments; $b$ ) ensure that recombinant DNA organisms are evaluated for potential risk, prior to applications in agriculture and the environment by means of an independent review of potential risks on a case-by-case basis; c) conduct the development of recombinant DNA organisms for agricultural or environmental applications in a stepwise fashion, moving, where appropriate, from the laboratory to the growth chamber and greenhouse, to limited field testing and finally, to large-scale field testing; d) encourage further research to improve the prediction, evaluation, and monitoring of the outcome of applications of recombinant DNA organisms." These recommendations lay down guiding principles for the risk evaluation of applications of genetically modified organisms that have remained valid: (a) the principle of familiarity, (b) the caseby-case approach, and (c) the step-by-step approach.

The appendices to the Blue Book provide guidance on how to apply the recommendations of the Blue Book in practice. They are probably the most widely-read and widely-used parts of the book. They have had a major impact on the development and practice of GMO risk assessment, as they have been used as a basic framework for the "recombinant DNA safety considerations" by probably all legislative systems that have been developed since the Blue Book.

\section{GOOD DEVELOPMENTAL PRINCIPLES (GDP) AND THE "TRAP OF GDP"}

After the publication of the Blue Book, safety issues were kept under review, within the OECD Group of National Experts on Safety in Biotechnology (GNE). This led to the publication of the "Safety considerations for biotechnology" (OECD, 1992). This publication presents a definition of "Good Developmental Principles" (GDP) for the design of safe small-scale field research with plants and micro-organisms with newly introduced traits. In order to be confident that the stepwise approach was feasible, it was a main prerequisite then that the first unconfined steps, i.e. small scale field testing, could indeed be performed in an environmentally safe way.
The paper clearly defines the stage in the development of a GMO where GDP applies (see Fig. 1): the early stages of field research.

The document stresses that it is expected that various control and safety measures, i.e. confinement, monitoring and/or mitigation measures, can be designed in such a way that small scale environmental can be performed at negligible risk. Ample examples of these control measures are provided in the document.

Logical as it may seem, the approach of GDP has also been criticized precisely for what it is aiming at: it secures that small-scale field experiments are set up in a safe way. If the results of such an experiment show that it was safe, nothing has been learned, except that the rules of GDP have been applied in the correct way! In reverse, the claim that the countless small scale field experiments that have been performed show that GM crops are safe is false for the same reason: as safety was already determined by the setup of these experiments, and cannot be proven by the experiments. This situation became known as the "trap of GDP", and a way out had to be found for it. The trap of GDP still occurs, when risk assessors claim that no risk assessment is needed for small scale "confined" field experiments, because the effects of the transgenic organisms are limited to the field trial, and the effects can be completely mitigated.

This is a formal breach of the order of steps in risk assessment: the determination of risk management measures is done after hazard identification (step 1), hazard assessment and exposure assessment (step 2) and risk evaluation (step 3). Only if an unacceptable risk is found, risk management measures have to be devised as step 4 . A practical consequence is that risk assessment is delayed to the next step, the "scale-up" of the field experiments. If hazard identification is only started at this step, valuable time may be lost if experiments necessary for hazard assessment still have to be done before scale-up can be started, while the experiments might have been performed during the small scale field experiments.

\section{CONSIDERATIONS FOR THE SCALE-UP OF CROP PLANTS}

The document "Safety considerations for biotechnology: scale-up of crop plants" (OECD, 1993a) was the result of extensive discussions of a working group set up within the GNE for drafting the document. Like the "Blue book" it brings together the views of experts with very different backgrounds. The aim of the document is to describe "how the concept of "familiarity" - with the plant, the introduced trait, the environment and their interaction may be applied to facilitate risk/safety analysis and to manage possible risks in the context of scaling up modified plants towards commercial release." 


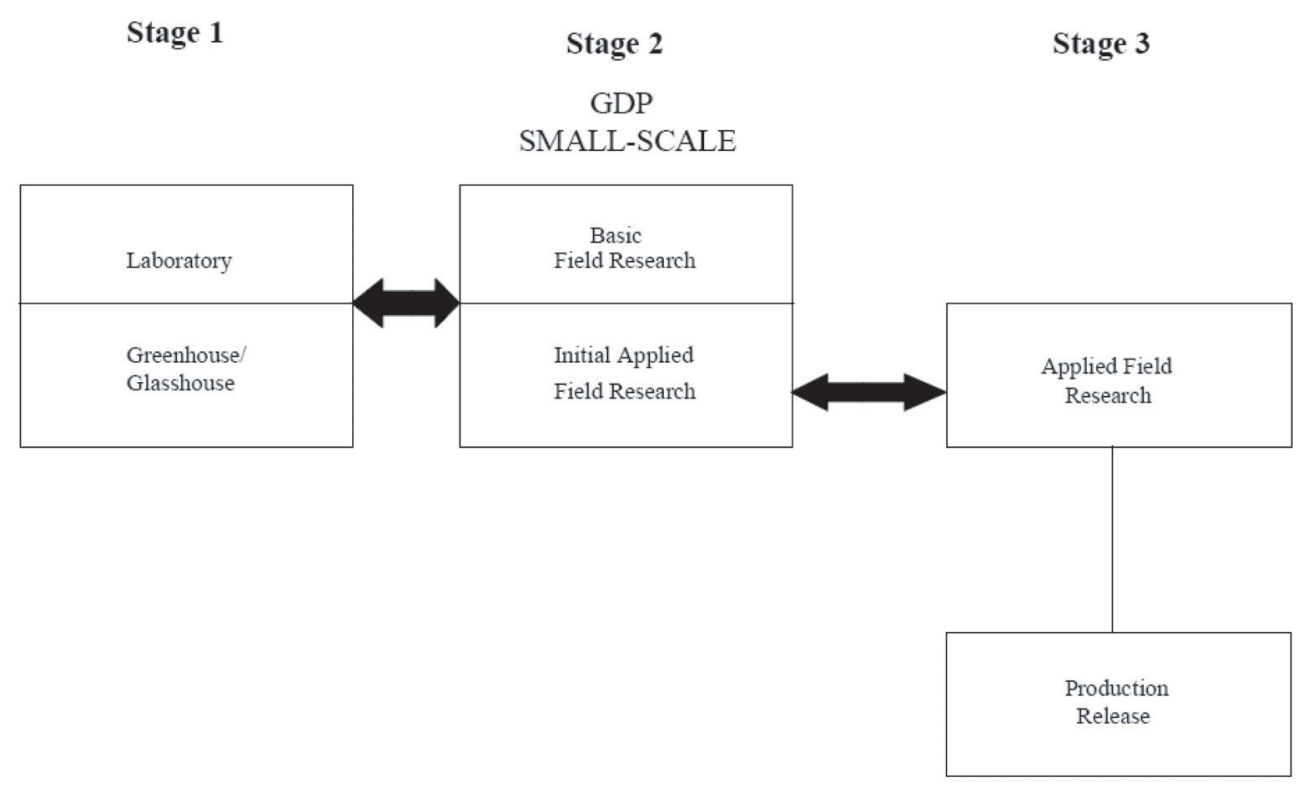

Figure 1. GDP in the context of field research.

The term "scale-up" is chosen on purpose, in preference to "large scale releases" to describe the continuum of research and development involving increasing scale from preliminary field tests up to general use. It is pointed out that scale-up for research purposes is not a new concept. Also in traditional plant breeding the process of cultivar development goes through a stage of performance trials where biosafety aspects of the new cultivar are screened, that are essentially similar to the safety issues of transgenic plants, e.g. any tendencies of specific lines "[...] to outcross, [...] to carry over at the site and become a weed, [...] to produce a known toxicant in the harvested product in quantities greater than the accepted standard, [...] to react/respond to other organisms in the environment, and any tendency for unwanted genetic or phenotypic variability beyond that observable during more preliminary evaluations in small-scale tests."

It should be remembered that these tendencies may be scale dependent, for instance because they occur at too low frequency to be detected in small-scale experiments. Some effects may only be detected when a cultivar is grown at commercial scale for several years. But, "this is normal and, based on familiarity, it is possible to predict, test, and plan for many events that may be of concern prior to wide-scale testing or general use."

The document then describes six safety concerns of a plant line with a new trait in a particular environment: "gene transfer, weediness, trait effects, genetic and phenotypic variability, biological vector effects and genetic material from pathogens, worker (human) safety", and goes into much detail how these concerns can be addressed during scale-up. "Familiarity", as the sum of knowledge and experience about a transgenic plant, or in fact about any factor considered in risk assessment, e.g. the host plant, the trait or the agronomic application, is a concept whose importance can hardly be overestimated. It should be stressed, however, that having familiarity is not a prerequisite for doing a risk assessment, neither is it equivalent to safety: some things that we are very familiar with pose severe risks.

\section{THE "PREAMBLE": THE LAY-OUT OF RISK ASSESSMENT}

At the time when the scale-up document was being written, there were very fundamental discussions within the GNE on the way risk analysis of transgenic organisms should be performed. As OECD is a consensus organization, the problems were approached by extensive exchange of views in many meetings of a subgroup of the GNE, leading to a publication that was intended to be added as a "preamble" to future documents on safety considerations for biotechnology. The preamble can be found for instance in the scale-up document (OECD, 1993a).

In the first place, the document speaks on purpose about risk/safety analysis, and states as a general principle that "safety in biotechnology is achieved by appropriate application of risk/safety analysis and risk management".

The document then very clearly states that "risk/safety analysis comprises hazard identification and if a hazard has been identified, risk assessment. 
Risk/safety analysis is based on the characteristics of the organism, the introduced trait, the interactions between these, and the intended application." The document then stresses the role of familiarity, and that where familiarity is lacking management practices may compensate.

The document makes an important point on how and when to do risk/safety analysis: it is "conducted prior to an intended action and is typically a routine and ongoing of research, development and testing of new organisms, whether performed in a laboratory or field setting. It ranges from a routine ad hoc judgment by the researcher as an implicit part of good experimental practices, to adherence to a formalized assessment. [It] is a scientific procedure which does not imply or exclude regulatory oversight or imply that every case will necessarily be reviewed by a national or other authority."

Many of the discussions around the "Preamble" focused on the concept of "step-by-step". It was felt by many that the concept as it is sometimes presented, i.e. as an obligation to go through defined steps in doing biosafety research, is not correct. To the contrary, the "concept of stepwise development and evaluation" implies that there is a "continuum of developmental stages" and that progression through the continuum is made based on information gathered from any empirical source, i.e. based on familiarity. It is the outcome of caseby-case risk/safety analysis at each stage that dictates the maximal extent of the next step that can be taken. Formalized steps may be too strict, when the risk/safety evaluation indicates that some of the steps may be skipped, but adherence to formalized steps may also be risky, in cases where risk/safety evaluation indicates that smaller steps are needed.

\section{THE CONCEPT OF FAMILIARITY: CONSENSUS DOCUMENTS}

While the scale-up document was being prepared, an effort was done to collate a body of knowledge and experience from "traditional crop breeding practices" (OECD, 1993b), that could, as the title says, "serve as a baseline for assessing the role of modern biotechnology".

The book starts with an introductory overview of plant breeding practice, that provides an outline of the research that is commonly done during variety development and testing, and that is relevant in the context of biosafety testing. The following chapters present the actual information for the major crops: soybean, wheat, rice, cucurbits, cotton, tobacco, sunflower, maize, sugar beet, alfalfa, oilseed rape, onion, cassava, potato and Prunus. This effort was done by individual experts in the field of agronomy.

The purpose of collating this knowledge is to provide "baseline data for use in risk evaluation use during the regulatory assessment of products of biotechnology" (OECD, 2005), i.e. to establish familiarity.

In 1995 the OECD Working Group on the Harmonization of Regulatory Oversight in Biotechnology was established. This Working Group took up the task to continue establishing familiarity in the fields that are important for risk assessment of transgenic organisms: establishing what is known about the biology of the recipient organisms, and about the physiology of the introduced traits.

The Working Group could, through its members, draw upon a large number of experts in all fields involved in biosafety of transgenic organisms, that could provide, validate and discuss the data that make up familiarity in this fields. The results of this process are being published in a series of OECD publications "on harmonization of oversight in biotechnology". These publications "are "snapshot" of current information, for use during the regulatory assessment of products of biotechnology. They are not intended to be a comprehensive source of information on everything that is known about a specific host organism or trait; but address - on a consensus basis the key or core set of issues that member countries believe are relevant to risk/ safety assessment". The "Introduction to the biosafety consensus documents of OECD's Working Group for Harmonisation in Biotechnology" (OECD, 2005) provides an excellent overview of the aims and development of these consensus documents.

An overview of the published consensus documents is available at http://www.oecd.org/document/ 51/0,2340,en_2649_34385_1889395_1_1_1_1,00.html.

They cover the common crop plants, including forest trees, some micro-organisms and a few traits, e.g. herbicide (glyphosate and phosphinothrycin) tolerance.

As the number of biology documents is constantly growing, it could be said that also "familiarity" is developing with drafting consensus documents. The Working Group therefore felt the need to develop a "structured explanatory checklist, regarding both order and contents, of relevant points to consider in preparing or evaluating a consensus document on the biology of a cultivated vascular plant species" (OECD, 2006).

\section{CONCLUSION}

In retrospect, the Blue Book has worked very efficiently as a thought starter for the further development of science-based risk/safety assessment, introducing concepts like GDP and familiarity. It sparked off the discussions that led to the drafting of consensus documents, i.e. the biology and trait documents. Much exchange of thought is still needed, particularly in the development of trait documents that for various reasons turn out to be 
more difficult to reach consensus on than the biology documents.

From this overview it should be apparent that while the documents reviewed here all have had enormous impact on the development of risk/safety assessment, none of them are "written in stone". They have mainly served their purpose in the discussions on risk assessment of transgenic organisms, and they deserve to be read still as such: to find out how the reasoning has developed over the years, and which arguments have been used when and why.

\section{REFERENCES}

Bull AT, Holt G, Lilly MD (1983) Biotechnology, international trends and perspectives. OECD, Paris
OECD (1986) Recombinant DNA safety considerations. OECD, Paris

OECD (1992) Safety considerations for biotechnology. OECD, Paris

OECD (1993a) Safety considerations for biotechnology: scaleup of crop plants. OECD, Paris

OECD (1993b) Traditional crop breeding practices: a historical review to serve as a baseline for assessing the role of modern biotechnology. OECD, Paris

OECD (2005) An introduction to the biosafety consensus documents of OECD's Working Group for Harmonisation in Biotechnology. OECD, Paris

OECD (2006) Points to consider for consensus documents on the biology of cultivated plants. OECD, Paris

All documents are available at http://www.oecd.org/ document/51/0,2340,en_2649_34385_1889395_1_1_1_1,00.html and

http://www.oecd.org/document/17/

0,2340,en_2649_34385_1891281_1_1_1_1,00.html 